\title{
FLOW OF REAL HYPERSURFACES BY THE TRACE OF THE LEVI FORM
}

\author{
Gerhard Huisken and Wilhelm Klingenberg
}

\section{Introduction}

Let $F_{0}: N^{2 n-1} \rightarrow \mathbb{C}^{n}, n \geq 2$, be a smooth immersion of a real $(2 n-1)$-dimensional hypersurface in complex $n$-space. In this paper we study the Cauchy-Riemann analogue of mean curvature flow $[\mathrm{H}]$ in Riemannian geometry: We deform the initial hypersurface $N_{0}=F_{0}\left(N^{2 n-1}\right)$ in normal direction such that the speed at each point is given by the trace of the Levi form of the induced CR-structure on the hypersurface, with the defining one-form of norm one. For simplicity we assume in this paper that $N^{2 n-1}$ is closed, i.e., compact without boundary. Our initial value problem for the flow along the trace of the Levi form then looks for a smooth family of immersions $F: N^{2 n-1} \times[0, T) \rightarrow \mathbb{C}^{n}$ satisfying the system

$$
\begin{aligned}
\frac{d}{d t} F(p, t) & =L(p, t) \cdot X_{2 n}(p, t), \\
F(p, 0) & =F_{0}(p),
\end{aligned}
$$

where $p \in N^{2 n-1}, t \in[0, T)$. Here $X_{2 n}(p, t)$ is the real unit normal of the hypersurface $N_{t}=F(\cdot, t)\left(N^{2 n-1}\right)$ at $F(p, t)$ in $\mathbb{C}^{n}$ (equipped with the standard euclidean metric) and $L(p, t)$ is the trace of the Levi form on $N_{t}$ at $F(p, t)$. On a closed hypersurface we choose $X_{2 n}$ to be the exterior unit normal and define the Levi form such that $\mathrm{L}$ is negative on the metric sphere in order to be consistent with the notation of $[\mathrm{K}]$.

This evolution equation is a weakly parabolic system exhibiting two different types of degeneracies: First, due to the invariance under tangential diffeomorphisms, the symbol is degenerate in tangential directions, a phenomenon which is well-known from mean curvature flow and can easily be dealt with by choosing a particular gauge.

Secondly and more seriously, the trace of the Levi form $L$ corresponds to a quasilinear second order differential operator ${ }^{H} \Delta$ on $N^{2 n-1}$ as in [K. 4.1], which acts like a Laplacian in complex directions $H N:=T N \cap \mathbb{C}^{n} J T N$ of $N$ but has a zero eigenvalue in its symbol in the direction of $T N$ that is orthogonal to $H N$.

After recalling the geometry of real hypersurfaces with their basic invariants from $[\mathrm{K}]$ in section 2 , we prove shorttime existence of solutions of (1.1) in section 3. The proof is based on uniform a priori estimates for the curvature and its

Received May 28, 1999. 
higher derivatives of solutions to approximating strictly parabolic systems. The necessary evolution equations for the curvature and its derivatives are computed with the help of commutator identities for the Kählerian shape operator established in $[\mathrm{K}]$. In addition to local existence we show that the solutions remain smooth as long as both the curvature and its first derivatives remain bounded. This result differs from standard mean curvature flow and is due to the degenerate parabolic structure of the flow. In section 4 we exploit the parabolic nature of (1.1) in establishing a barrier principle for distinct solutions of the flow. We also show that embedded solutions stay embedded. In the case $n=2$, where the Levi form reduces to a real scalar function, we prove a version of the strict maximum principle to show that smooth, closed weakly pseudoconvex surfaces instantaneously become strictly pseudoconvex under the evolution (1.1). This provides a canonical approximation of such weakly pseudoconvex surfaces by strictly pseudoconvex surfaces. We expect stronger regularity properties of the flow in the strictly pseudoconvex case, which will be examined in a future article. We refer to $[\mathrm{ST}]$ for a weak solution of (1.1) in the level set formulation.

Acknowledgement. We are grateful to Mrs. S. Schmidt for typing the manuscript.

Remark. We wish to draw the attention of the reader to the recent work $[\mathrm{C}]$ and $[\mathrm{CM}]$.

\section{Notation and preliminaries}

Here we review the notation and results in $[\mathrm{K}]$ that will be used throughout this paper. For a real hypersurface $F: N \rightarrow \mathbb{C}^{n}$ let $H N=T V \cap \mathbb{C}^{n}$ JTN be the maximal complex tangent bundle in $T N$. We denote by $T^{1,0}$ and $T^{0,1}$ the eigenspaces for $i$ and $-i$ of $\mathbb{C} \otimes T \mathbb{C}^{n}$. The Kählerian geometry of a hypersurface can be extracted from the principle bundle of unitary frames $\left\{e_{j}\right\}_{1}^{n}$ of $F^{*} T^{1,0} \mathbb{C}^{n}$ that are adapted to $T N \hookrightarrow F^{*} T \mathbb{C}^{n}$ in the following way: $\operatorname{span}\left\{e_{\alpha}\right\}_{1}^{n-1}=H^{1,0} N$, $\operatorname{span}\left\{f_{\alpha}:=e_{\alpha}, f_{\bar{\alpha}}:=e_{\bar{\alpha}}, f_{n}:=\frac{1}{2}\left(e_{n}+e_{\bar{n}}\right)\right\}=\mathbb{C} \otimes T N$. We set $X_{j}=\frac{1}{\sqrt{2}}\left(e_{j}+e_{\bar{j}}\right)$, $X_{n+j}=\frac{i}{\sqrt{2}}\left(e_{j}-e_{\bar{j}}\right)$. It follows that $X_{2 n}$ is normal to $T N$. We have the following convention for ranges of indices: $j, k, p, q \in\{1, \ldots, n\}, \alpha, \beta, \gamma \in\{1, \ldots, n-1\}$, $\xi, \eta \in\{1, \ldots, n-1, \overline{1}, \ldots, \overline{n-1}\}, a, b, c \in\{1, \ldots, n, \overline{1}, \ldots, \bar{n}\}$. It follows for example that $\operatorname{span}\left\{f_{\alpha}\right\}=H^{1,0} N, \operatorname{span}\left\{f_{\xi}\right\}=\mathbb{C} \otimes H N, \operatorname{span}\left\{f_{a}\right\}=\mathbb{C} \otimes T N$. On the bundles $H N \hookrightarrow T N \hookrightarrow F^{*} T N$ over $N$ we have the Levi-Civita connection $\mathbb{C}^{n} \nabla$ and its projections ${ }^{N} \nabla,{ }^{H} \nabla$ with connection forms ${ }^{C^{n}} \omega,{ }^{N} \omega,{ }^{H} \omega$ and curvature forms ${ }^{\mathbb{C}^{n}} \Omega,{ }^{N} \Omega,{ }^{H} \Omega$; see $[\mathrm{K}, 2$.$] for their structure equations and$ interrelations. In $[\mathrm{K}, 1$.$] Klingenberg defines a tensor \ell$ on $\mathbb{C} \otimes T N$ that takes the role of a Kählerian shape operator of $N \rightarrow \mathbb{C}^{n}$ :

$$
\ell_{j a}:=\theta^{n}\left(\mathbb{C}^{n} \nabla_{f_{a}} e_{j}\right), \quad j=1, \ldots, n, \quad a=1, \ldots, n-1, \overline{1}, \ldots, \overline{n-1}, n .
$$

Here, $\left\{\theta^{j}\right\}$ is the dual frame of $\left\{e_{j}\right\},\left\{\ell_{\alpha \bar{\beta}}\right\}_{1}^{n-1}$ is the Levi form of $N \rightarrow \mathbb{C}^{n}$, which is hermitian on $H^{1,0} N \otimes H^{0,1} N,\left\{\ell_{\alpha \beta}\right\}$ is symmetric on $H^{1,0} N \otimes H^{1,0} N$, 
and $\left\{\ell_{n a}\right\}$ is a real form on $\mathbb{C} \otimes T N$. We will also denote by $\left\{\xi^{j}, \xi^{n+j}\right\}$ the dual frame of $\left\{X_{j}, X_{n+j}\right\}$ and $\left\{\varphi^{a}\right\}$ the dual frame of $\left\{f_{a}\right\}$. The trace of the Levi form is given by

$$
L:=\Sigma_{1}^{n-1} \ell_{\alpha \bar{\alpha}}
$$

It equals the negative trace of the restriction of the Riemannian second fundamental form to $H N \hookrightarrow T N$, see [K, 1.4]. In addition, the total norm $|A|^{2}$ of the Riemannian second fundamental form is controlled by the total norm of $\ell_{j a}$ :

$$
|A|^{2} \leq 2|\ell|^{2}, \quad|\ell|^{2}:=\Sigma_{j a}\left|\ell_{j a}\right|^{2} .
$$

We also set $\ell_{a b}^{2}=\ell_{a \bar{\gamma}} \ell_{\gamma b}$ and $\ell_{a b}^{3}=\ell_{a \bar{\gamma}} \ell_{\gamma \bar{\delta}} \ell_{\delta b}$. One can always choose a unitary frame $\left\{e_{\alpha}\right\}$ of $H^{1,0} N$ so that $\ell_{\alpha \bar{\beta}}=0$ for $\alpha \neq \beta$ at a given point $p \in N$. We denote by $\ell_{j a b}^{H}$ and $\ell_{j a b}^{N}$ the components of the first covariant derivative of $\ell$ with respect to the connection on $H N$ and $T N$. Finally we note that with the notation

$$
{ }^{H} \Delta={ }^{H} \nabla_{e_{\xi}}{ }^{H} \nabla_{e_{\bar{\xi}}}-{ }^{H} \nabla^{H} \nabla_{e_{\xi}} e_{\bar{\xi}} \equiv \operatorname{trace}_{H}\left({ }^{H} \nabla^{2}\right),
$$

(1.1) has the form

$$
\frac{d}{d t} F(p, t)={ }^{H} \Delta F(p, t),
$$

which is analogous to the mean curvature flow in $[\mathrm{H}]$ given by

$$
\frac{d}{d t} F(p, t)={ }^{N} \Delta F(p, t) .
$$

\section{Short time existence}

The aim of this section is to establish two main existence results. First we prove short time existence of a solution of (1.1) with smooth initial data.

Theorem 3.1. Given a smooth regular immersion $F_{0}: N^{2 n-1} \rightarrow \mathbb{C}^{n}$ of a closed hypersurface in complex Euclidean space, there exists a time interval $\left[0, t_{0}\right)$, $t_{0}>0$, with a smooth regular solution $F: N^{2 n-1} \times\left[0, t_{0}\right) \rightarrow \mathbb{C}^{n}$ of (1.1).

We can also show that the solution will continue to exist as long as the total curvature and the total gradient of the evolving hypersurface remains bounded. Notice that this result is in contrast to the mean curvature flow $[\mathrm{H}]$, where only the curvature needs to be bounded to establish longtime existence. The stronger condition here is due to the degeneracy in this flow which inhibits diffusion in the direction normal to $H N$. We expect a better regularity result in the strictly pseudoconvex case.

Theorem 3.2. There exists a maximal time interval $[0, T), T>0$, admitting a smooth regular solution of (1.1). As $t \rightarrow T$, the total curvature $\sup _{N_{t}}|\ell|^{2}(t)$ or its total derivative $\sup _{N_{t}}|\nabla \ell|^{2}(t)$ becomes unbounded. 
Remarks: i) The a priori estimates for the proof of Theorem 3.1 will also yield a lower bound for $T$ in terms of the initial curvature and initial gradient of curvature, see Corollary 3.6.

ii) We will show in section 4 that for compact initial data $T$ is bounded above in terms of the radius $R_{0}$ of the smallest sphere containing $N_{0}: T \leq R_{0}^{2} / 4(n-1)$. iii) Since equation (1.1) is more degenerate than mean curvature flow, we cannot expect a general smoothing behaviour like in that case and Theorem 3.1 depends heavily on the smoothness of the initial data. However, it will become apparent in the proof that for intitial data in $C^{k-1,1}, k \geq 3$, a solution exists in $C^{k-1,1}$. Whether some smoothing result is true, e.g., for strictly pseudoconvex initial data, is an interesting open problem.

iv) Theorems 3.1 and 3.2 can be extended to hypersurfaces in arbitrary Kähler manifolds, since the corresponding evolution equations for $\ell_{j a}$ differ only by terms involving the given curvature tensor of the ambient manifold and are of lower order in the estimates derived here.

To prove the two theorems we first consider smooth regular solutions of the evolution equation for a regular $F: N \times[0, T) \rightarrow \mathbb{C}^{n}$ :

$$
\frac{d}{d t} F=K X_{2 n} \quad \text { on } \quad N^{2 n-1} \times[0, T),
$$

with an arbitrary speed function $K$, and establish how the curvature changes under this flow. Given an adapted frame $\left\{e_{k}\right\}$ of $F^{*}(\cdot, 0) T^{1,0} \mathbb{C}^{n}$, we need to define its time evolution in $F^{*}(\cdot, t) T^{1,0} \mathbb{C}^{n}$ over $N_{t}$. For that we extend $F$ to a map $\tilde{F}: N \times[0, T) \rightarrow \mathbb{C}^{n} \times \mathbb{R}, \tilde{F}(p, t)=(F(p, t), t)$. Then the bundle $\tilde{F}^{*} T\left(\mathbb{C}^{n} \times \mathbb{R}\right)$ $=F^{*} T \mathbb{C}^{n} \oplus \mathbb{R}$ over $N \times[0, T)$ can be equipped with the product metric in each fibre and the Levi-Civita connection $\mathbb{C}^{n} \times \mathbb{R} \nabla$. In addition, $\left\{e_{k}\right\}$ is naturally a frame of $\tilde{F}^{*}(\cdot, 0)\left(T^{1,0} \mathbb{C}^{n} \oplus 0\right)=F^{*}(\cdot, 0) T^{1,0} \mathbb{C}^{n} \oplus 0$ over $N_{0} \times 0$. Extend ${ }^{\mathbb{C}^{n} \times \mathbb{R}} \nabla$ trivially to $T \tilde{F}^{*}(\cdot, 0)\left(T \mathbb{C}^{n} \oplus \mathbb{R}\right)=F^{*}\left(T \mathbb{C}^{n} \oplus \mathbb{R}\right) \oplus \mathbb{C}^{n} \oplus \mathbb{R}$ and set

$$
Z:=\tilde{F}^{*} \tilde{F}_{*}(\partial / \partial t)
$$

Then $Z=\left(K X_{2 n}, 1\right)$ is transverse to $N \times t$ for every $t$. Setting $K_{a}:=f_{a} \cdot K$, the following system of ODEs then uniquely determines an evolution for $\left\{e_{k}\right\}$ :

$$
\begin{aligned}
& \nabla_{Z} e_{\alpha}=i \sqrt{2} K_{\alpha} e_{n}, \\
& \nabla_{Z} e_{n}=i \sqrt{2} K_{j} e_{j} .
\end{aligned}
$$

This is well posed since for every $t$, the vector $Z$ is transverse to $N \times t$ in $T \tilde{F}^{*}\left(T \mathbb{C}^{n} \oplus 0\right)$. In addition by the Kähler property, this evolution gives a frame of $\tilde{F}^{*}(\cdot, t)\left(T^{1,0} \mathbb{C}^{n} \oplus 0\right)$. We finally extend the frame by parallel transport along the vectorfield $X_{2 n}$ to

$T \tilde{F}^{*}\left(T^{1,0} \mathbb{C}^{n} \oplus 0\right)$. This implies that $\nabla_{Z} e_{j}=\nabla_{\frac{\partial}{\partial t}} e_{j}$ and we will often simply write $\frac{\partial}{\partial t}$ to denote such a derivative. We claim that the above evolution implies

$$
\begin{aligned}
\xi^{2 n}\left(\mathcal{L}_{Z} f_{a}\right) & =0, \\
\theta^{\beta}\left({ }^{\mathbb{C}^{n} \times \mathbb{R}} \nabla_{Z} e_{\alpha}\right) & =0 .
\end{aligned}
$$


Thus our choice of evolution for the frame $\left\{e_{j}\right\}$ ensures that the frame remains adapted to $H N_{t} \subset T N_{t}$, without rotating the holomorphic directions. To prove the claim, note that (3.3) implies $\nabla_{\partial / \partial t} X_{2 n}=-K_{\xi} f_{\bar{\xi}}-2 K_{n} f_{n}$, and therefore $\varphi^{n}\left(\nabla_{\partial / \partial t} X_{2 n}\right)=-2 K_{n}, \varphi^{\bar{\xi}}\left(\nabla_{\partial / \partial t} X_{2 n}\right)=-K_{\xi}$, which gives $\xi^{2 n}\left(\nabla_{\partial / \partial t} f_{a}\right)=K_{a}$ and implies (3.4). Also, (3.2) clearly implies (3.5). This proves the claim. In addition, we infer the identities

$$
\begin{aligned}
\mathcal{L}_{\partial / \partial t} f_{\alpha} & =\frac{1}{2} K \ell_{\alpha \bar{\xi}} f_{\xi}+\left(K \ell_{\alpha n}+i \sqrt{2} K_{\alpha}\right) f_{n} \\
\mathcal{L}_{\partial / \partial t} f_{n} & =\frac{1}{2} K \ell_{n \bar{\xi}} f_{\xi}+\frac{i}{\sqrt{2}}\left(K_{\bar{\gamma}} f_{\gamma}-K_{\gamma} f_{\bar{\gamma}}\right)+K \ell_{n n} f_{n} .
\end{aligned}
$$

Proposition 3.3. For the evolution (3.1) of hypersurfaces, we have the following evolution of $\ell$ with respect to the frame constructed above.

$$
\begin{aligned}
& \frac{\partial}{\partial t} \ell_{\alpha \xi}= 2\left({ }^{H} \nabla^{2} K\right)\left(f_{\alpha}, f_{\xi}\right)+i \sqrt{2}\left(\ell_{n \xi} K_{\alpha} \pm \ell_{n \alpha} K_{\xi}-\ell_{\alpha \xi} K_{n}\right) \\
&+ \frac{1}{2} K \ell_{\alpha \bar{\eta}} \ell_{\eta \xi}+K \ell_{\alpha n} \ell_{n \xi}, \\
& \frac{\partial}{\partial t} \ell_{n \alpha}=2\left({ }^{H} \nabla^{2} K\right)\left(f_{n}, f_{\alpha}\right)+i \sqrt{2}\left(\ell_{\alpha \gamma} K_{\bar{\gamma}}-\ell_{\alpha \bar{\gamma}} K_{\gamma}+\ell_{n n} K_{\alpha}-\ell_{\alpha n} K_{n}\right)+\frac{1}{2} K \ell_{\alpha \bar{\eta}} \ell_{\eta n}+K \ell_{\alpha n} \ell_{n n}, \\
& \frac{\partial}{\partial t} \ell_{n n}=2\left({ }^{H} \nabla^{2} K\right)\left(f_{n}, f_{n}\right)+\frac{3 i}{\sqrt{2}}\left(\ell_{n \gamma} K_{\bar{\gamma}}-\ell_{n \bar{\gamma}} K_{\gamma}\right) \\
&+\frac{1}{2} K \ell_{n \bar{\eta}} \ell_{\eta n}+K\left(\ell_{n n}\right)^{2} .
\end{aligned}
$$

In the first equation, the upper sign holds if $\xi \in\{1, \ldots, n-1\}$ and the lower sign holds otherwise.

Remark: It is easy to see that the evolution equations above only depend on the construction of the frame on $N \times[0, T)$, not on the parallel extension of the frame along $X_{2 n}$ in the ambient space. For a different extension of the frame to the ambient space the time derivative is then replaced by differentiation in direction $Z$.

Proof. By the product rule,

$$
\frac{\partial}{\partial t} \theta^{n}\left(\nabla_{f_{a}} e_{j}\right)=\left(\nabla_{\partial / \partial t} \theta^{n}\right)\left(\nabla_{f_{a}} e_{j}\right)+\theta^{n}\left(\nabla_{\left[\partial / \partial t, f_{a}\right]} e_{j}\right)+\theta^{n}\left(\nabla_{f_{a}} \nabla_{\partial / \partial t} f_{a}\right) .
$$

Using the relations (3.2)-(3.7) and the identities $f_{a} \cdot K_{\alpha}=\left({ }^{H} \nabla^{2} K\right)\left(f_{\alpha}, f_{a}\right) K+$ $K_{\delta} \omega_{\alpha}^{\delta}\left(f_{a}\right), f_{a} \cdot K_{n}=\left({ }^{H} \nabla^{2} K\right)\left(f_{n}, f_{a}\right)$, we compute the above expression for 
various $(a, j)$.

$$
\begin{aligned}
& \frac{\partial}{\partial t} \theta^{n}\left(\nabla_{f_{\xi}} e_{\alpha}\right)=-i \sqrt{2} K_{j} \theta^{j}\left(\nabla_{f_{\xi}} e_{\alpha}\right)+\frac{1}{2} K \ell_{\xi \bar{\eta}} \theta^{n}\left(\nabla_{f_{\eta}} e_{\alpha}\right) \\
& +\left(K \ell_{n \xi} \pm i \sqrt{2} K_{\xi}\right) \theta^{n}\left(\nabla_{f_{n}} e_{\alpha}\right) \\
& +i \sqrt{2} K_{\alpha} \theta^{n}\left(\nabla_{f_{\xi}} e_{n}\right)+i \sqrt{2}\left({ }^{H} \nabla^{2} K\right)\left(f_{\alpha}, f_{\xi}\right) \\
& +i \sqrt{2} K_{\delta} \omega_{\alpha}^{\delta}\left(f_{\xi}\right) \\
& =-i \sqrt{2} K_{n} \frac{i}{\sqrt{2}} \ell_{\alpha \xi}+\frac{i}{2^{3 / 2}} K \ell_{\xi \bar{\eta}} \ell_{\eta \alpha} \\
& +\frac{i}{\sqrt{2}} K \ell_{n \xi} \ell_{n \alpha} \mp \ell_{n \alpha} K_{\xi}-\ell_{n \xi} K_{\alpha} \\
& +i \sqrt{2}\left({ }^{H} \nabla^{2} K\right)\left(f_{\alpha}, f_{\xi}\right), \\
& \frac{\partial}{\partial t} \theta^{n}\left(\nabla_{f_{\alpha}} e_{n}\right)=-i \sqrt{2} K_{j} \theta^{j}\left(\nabla_{f_{\alpha}} e_{n}\right)+\frac{1}{2} K \ell_{\alpha \bar{\xi}} \theta^{n}\left(\nabla_{f_{\xi}} e_{n}\right) \\
& +\left(K \ell_{\alpha n}+i \sqrt{2} K_{\alpha}\right) \theta^{n}\left(\nabla_{f_{n}} e_{n}\right)+i \sqrt{2} K_{\bar{j}} \theta^{n}\left(\nabla_{f_{\alpha}} e_{j}\right) \\
& +i \sqrt{2}\left({ }^{H} \nabla^{2} K\right)\left(f_{n}, f_{\alpha}\right) \\
& =K_{\gamma} \ell_{\alpha \bar{\gamma}}+\frac{i}{2^{3 / 2}} K \ell_{\alpha \bar{\xi}} \ell_{\xi n}+\frac{i}{\sqrt{2}} K \ell_{\alpha n} \ell_{n n} \\
& -K_{\alpha} \ell_{n n}-K_{\bar{\gamma}} \ell_{\alpha \gamma}+i \sqrt{2}\left({ }^{H} \nabla^{2} K\right)\left(f_{n}, f_{\alpha}\right), \\
& \frac{\partial}{\partial t} \theta^{n}\left(\nabla_{f_{n}} e_{n}\right)=-i \sqrt{2} K_{j} \theta^{j}\left(\nabla_{f_{n}} e_{n}\right)+\frac{1}{2} K \ell_{n \bar{\eta}} \theta^{n}\left(\nabla_{f_{\eta}} e_{n}\right) \\
& +\frac{i}{\sqrt{2}}\left(K_{\bar{\gamma}} \theta^{n}\left(\nabla_{f_{\gamma}} e_{n}\right)-K_{\gamma} \theta^{n}\left(\nabla_{f_{\bar{\gamma}}} e_{n}\right)\right) \\
& +K \ell_{n n} \theta^{n}\left(\nabla_{f_{n}} e_{n}\right)+i \sqrt{2}\left({ }^{H} \nabla^{2} K\right)\left(f_{n}, f_{n}\right) \\
& +i \sqrt{2} K_{\bar{j}} \theta^{n}\left(\nabla_{f_{n}} e_{j}\right) \\
& =K_{\gamma} \ell_{n \bar{\gamma}}+\frac{i}{2^{3 / 2}} K \ell_{n \bar{\eta}} \ell_{\eta n}-\frac{1}{2} K_{\bar{\gamma}} \ell_{\gamma n} \\
& +\frac{1}{2} K_{\gamma} \ell_{n \bar{\gamma}}+\frac{i}{\sqrt{2}} K\left(\ell_{n n}\right)^{2}+i \sqrt{2}\left({ }^{H} \nabla^{2} K\right)\left(f_{n}, f_{n}\right) \\
& -K_{\bar{\gamma}} \ell_{n \gamma} \text {. }
\end{aligned}
$$

These easily imply the claims.

We will apply Theorem 3.1 with $K=L+\varepsilon L^{N}$, where $-L^{N}$ is the Riemannian mean curvature of the hypersurface. The parabolic nature of the equations in Theorem 3.1 will become apparent by combining them with the commutator equations established in $[\mathrm{K}, 4.2]$. 
Proposition 3.4. Let $\square_{\varepsilon}=\frac{\partial}{\partial t}-\left({ }^{H} \Delta+\varepsilon^{N} \Delta\right)$. Then

$$
\begin{aligned}
& \square_{\varepsilon} \ell_{\alpha \bar{\beta}}=i \sqrt{2}\left[\ell_{n \bar{\gamma}} \ell_{\alpha \bar{\beta} \gamma}^{H}-\ell_{n \gamma} \ell_{\alpha \bar{\beta} \bar{\gamma}}^{H}+2\left(\ell_{\gamma \bar{\beta}} \ell_{\alpha \bar{\gamma} n}^{H}-\ell_{\alpha \bar{\gamma}} \ell_{\gamma \bar{\beta} n}^{H}\right)\right] \\
& +\frac{1}{2} \ell_{\alpha \bar{\beta}}\left(\ell_{\gamma \bar{\gamma}}^{2}+\ell_{\bar{\gamma} \gamma}^{2}+2 \ell_{n n}^{2}\right)+\frac{1}{2}\left(\ell_{\alpha \gamma}^{2} \ell_{\bar{\gamma} \bar{\beta}}+\ell_{\alpha \gamma} \ell_{\bar{\gamma} \bar{\beta}}^{2}\right)-\ell_{\bar{\beta} \alpha}^{3} \\
& +\varepsilon\left[i \sqrt{2}\left(\ell_{n \bar{\beta}} L_{\alpha}^{N}-\ell_{n \alpha} L_{\bar{\beta}}^{N}\right)+|\ell|^{2} \ell_{\alpha \bar{\beta}}\right], \\
& \left.\square_{\varepsilon} \ell_{\alpha \beta}=i \sqrt{2}\left[\ell_{n \bar{\gamma}} \ell_{\alpha \beta \gamma}^{H}-\ell_{n \gamma} \ell_{\alpha \beta \bar{\gamma}}^{H}-2 \ell_{\alpha \bar{\gamma}} \ell_{\gamma \beta n}^{H}+\ell_{\beta \bar{\gamma}}^{2} \ell_{\gamma \alpha n}^{H}\right)\right] \\
& +\frac{1}{2} \ell_{\alpha \beta}\left(\ell_{\gamma \bar{\gamma}}^{2}+\ell_{\bar{\gamma} \gamma}^{2}+2 \ell_{n n}^{2}\right)+2 \ell_{n n}\left(\ell_{\alpha \beta}^{2}+\ell_{\beta \alpha}^{2}\right) \\
& -4\left(\ell_{\alpha n}^{2} \ell_{n \beta}+\ell_{\alpha n} \ell_{\beta n}^{2}\right)+\frac{1}{2}\left(\ell_{\alpha \gamma} \ell_{\bar{\gamma} \beta}^{2}+\ell_{\bar{\gamma} \alpha}^{2} \ell_{\gamma \beta}\right)-\frac{1}{2}\left(\ell_{\alpha \beta}^{3}+\ell_{\beta \alpha}^{3}\right) \\
& +\varepsilon\left[i \sqrt{2}\left(\ell_{n \beta} L_{\alpha}^{N}+\ell_{n \alpha} L_{\beta}^{N}\right)+|\ell|^{2} \ell_{\alpha \beta}\right], \\
& \square_{\varepsilon} \ell_{n \alpha}=i \sqrt{2}\left[-2 \ell_{\alpha \bar{\gamma}} \ell_{\gamma n n}^{H}+\frac{1}{2}\left(\ell_{\gamma \delta} \ell_{\bar{\gamma} \bar{\delta} \alpha}^{H}-\ell_{\bar{\gamma} \bar{\delta}} \ell_{\gamma \delta \alpha}^{H}\right)-\ell_{\alpha n} L_{n}\right] \\
& +\ell_{n \alpha}\left(\ell_{\gamma \bar{\gamma}}^{2}+\ell_{\bar{\gamma} \gamma}^{2}\right)-2\left(\ell_{\alpha \bar{\gamma}} \ell_{n \gamma}^{2}+\ell_{n \alpha}^{3}\right)+\frac{1}{2}\left(\ell_{n \gamma} \ell_{\bar{\gamma} \alpha}^{2}+\ell_{\alpha n}^{3}\right) \\
& +\varepsilon\left[i \sqrt{2}\left(\ell_{\alpha \gamma} L_{\bar{\gamma}}^{N}-\ell_{\alpha \bar{\gamma}} L_{\gamma}^{N}+\ell_{n n} L_{\alpha}^{N}\right)+|\ell|^{2} \ell_{\alpha n}\right], \\
& \square_{\varepsilon} \ell_{n n}=i \sqrt{2}\left(\ell_{n \gamma} \ell_{n n \bar{\gamma}}-\ell_{n \bar{\gamma}} \ell_{n n \gamma}+\ell_{\gamma \delta} \ell_{\bar{\gamma} \bar{\delta} n}-\ell_{\bar{\gamma} \bar{\delta}} \ell_{\gamma \delta n}\right) \\
& +\frac{3}{2} \ell_{n n}\left(\ell_{\gamma \bar{\gamma}}^{2}+\ell_{\bar{\gamma} \gamma}^{2}+\frac{3}{2} \ell_{n n}^{2}\right)-6 \ell_{n n}^{3}+\frac{1}{2}\left(\ell_{\gamma \bar{\delta}} \ell_{\bar{\gamma} \delta}^{2}-\ell_{\gamma \bar{\gamma}}^{3}\right) \\
& +\varepsilon\left[\frac{3 i}{\sqrt{2}}\left(\ell_{n \gamma} L_{\bar{\gamma}}^{N}-\ell_{n \bar{\gamma}} L_{\gamma}^{N}+\frac{2}{3} \ell_{n n} L_{n}^{N}\right)+|\ell|^{2} \ell_{n n}\right] .
\end{aligned}
$$

Proof. Note that by definition of ${ }^{N} \nabla$ and ${ }^{H} \nabla$, as explained in section 2, $\left({ }^{N} \nabla^{2} L^{N}\right)\left(f_{j}, f_{a}\right)+L_{n}^{N}{ }^{N} \omega_{j}^{n}\left(f_{a}\right)=\left({ }^{H} \nabla^{2} L^{N}\right)\left(f_{j}, f_{a}\right)$. By [K, 2.1] we have ${ }^{N} \omega_{j}^{n}\left(f_{a}\right)=\omega_{j}^{n}\left(f_{a}\right)$ such that in view of the commutator identities from $[\mathrm{K}$, $4.0,4.2]$ for ${ }^{N} \Delta,{ }^{H} \Delta$ we may proceed as follows with $K=L+\varepsilon L^{N}$. In this computation we use Proposition 3.1 and the definition of $\ell_{(a b)}^{2}$ given in [K, 4.0].

$$
\begin{gathered}
\square_{\varepsilon} \ell_{\alpha \bar{\beta}}=\left(\frac{\partial}{\partial t} \ell_{\alpha \bar{\beta}}-2\left({ }^{H} \nabla^{2} K\right)\left(f_{\alpha}, f_{\bar{\beta}}\right)\right)+\left(2\left({ }^{H} \nabla^{2} L\right)\left(f_{\alpha}, f_{\bar{\beta}}\right)-{ }^{H} \Delta \ell_{\alpha \bar{\beta}}\right) \\
+\varepsilon\left(2\left({ }^{N} \nabla^{2} L^{N}\right)\left(f_{\alpha}, f_{\bar{\beta}}\right)+2 L_{n}^{N} \omega_{\alpha}^{n}\left(f_{\bar{\beta}}\right)-{ }^{N} \Delta \ell_{\alpha \bar{\beta}}\right) \\
=i \sqrt{2}\left(\ell_{n \bar{\beta}} K_{\alpha}-\ell_{n \alpha} K_{\bar{\beta}}-\ell_{\alpha \bar{\beta}} K_{n}\right)+\frac{1}{2} K \ell_{\alpha \bar{\eta}} \ell_{\eta \bar{\beta}} \\
+K \ell_{\alpha n} \ell_{n \bar{\beta}}+i \sqrt{2}\left[\ell_{n \bar{\gamma}} \ell_{\alpha \bar{\beta} \gamma}^{H}-\ell_{n \gamma} \ell_{\alpha \bar{\beta} \bar{\gamma}}^{H}\right. \\
+2\left(\ell_{\gamma \bar{\beta}} \ell_{\alpha \bar{\gamma} n}^{H}-\ell_{\alpha \bar{\gamma}} \ell_{\gamma \bar{\beta} n}^{H}\right)+\ell_{n \alpha} L_{\bar{\beta}} \\
\left.-\ell_{n \bar{\beta}} L_{\alpha}+\ell_{\alpha \bar{\beta}} L_{n}\right]
\end{gathered}
$$




$$
\begin{aligned}
& +\frac{1}{2} \ell_{\alpha \bar{\beta}}\left(\ell_{\gamma \bar{\gamma}}^{2}+\ell_{\bar{\gamma} \gamma}^{2}+2 \ell_{n n}^{2}\right)-\frac{1}{2} L\left(\ell_{\alpha \bar{\beta}}^{2}+\ell_{\bar{\beta} \alpha}^{2}+2 \ell_{n \alpha} \ell_{n \bar{\beta}}\right) \\
& +\frac{1}{2}\left(\ell_{\alpha \gamma}^{2} \ell_{\bar{\gamma} \bar{\beta}}+\ell_{\alpha \gamma} \ell_{\bar{\gamma} \bar{\beta}}^{2}\right)-\ell_{\bar{\beta} \alpha}^{3} \\
& +\varepsilon\left(|\ell|^{2} \ell_{\alpha \bar{\beta}}-L^{N} \ell_{(\alpha \bar{\beta})}^{2}+i \sqrt{2} \ell_{\alpha \bar{\beta}}-L_{n}^{N}\right), \\
& \square_{\varepsilon} \ell_{\alpha \beta}=\left(\frac{\partial}{\partial t} \ell_{\alpha \beta}-2\left({ }^{H} \nabla^{2} K\right)\left(f_{\alpha}, f_{\beta}\right)\right)+\left(2\left({ }^{H} \nabla^{2} L\right)\left(f_{\alpha}, f_{\beta}\right)-{ }^{H} \Delta \ell_{\alpha \beta}\right) \\
& +\varepsilon\left(2\left({ }^{N} \nabla^{2} L^{N}\right)\left(f_{\alpha}, f_{\beta}\right)+2 L_{n}^{N}{ }^{N} \omega_{\alpha}^{n}\left(f_{\beta}\right)-{ }^{N} \Delta \ell_{\alpha \beta}\right) \\
& =i \sqrt{2}\left(\ell_{n \beta} K_{\alpha}+\ell_{n \alpha} K_{\beta}-\ell_{\alpha \beta} K_{n}\right)+\frac{1}{2} K \ell_{\alpha \bar{\eta}} \ell_{\eta \beta} \\
& +K \ell_{\alpha n} \ell_{\beta n}+i \sqrt{2}\left[\ell_{n \bar{\gamma}} \ell_{\alpha \beta \gamma}^{H}-\ell_{n \gamma} \ell_{\alpha \beta \bar{\gamma}}^{H}\right. \\
& -2\left(\ell_{\alpha \bar{\gamma}} \ell_{\gamma \beta n}^{H}+\ell_{\beta \bar{\gamma}} \ell_{\gamma \alpha n}^{H}\right) \\
& \left.-\ell_{n \alpha} L_{\beta}-\ell_{n \beta} L_{\alpha}+\ell_{\alpha \beta} L_{n}\right] \\
& +\frac{1}{2} \ell_{\alpha \beta}\left(\ell_{\gamma \bar{\gamma}}^{2}+\ell_{\bar{\gamma} \gamma}^{2}+2 \ell_{n n}^{2}\right) \\
& -\frac{1}{2} L\left(\ell_{\alpha \beta}^{2}+\ell_{\beta \alpha}^{2}+2 \ell_{\alpha n} \ell_{\beta n}\right)+2 \ell_{n n}\left(\ell_{\alpha \beta}^{2}+\ell_{\beta \alpha}^{2}\right) \\
& -4\left(\ell_{\alpha n}^{2} \ell_{n \beta}+\ell_{\alpha n} \ell_{\beta n}^{2}\right)+\frac{1}{2}\left(\ell_{\alpha \gamma} \ell_{\bar{\gamma} \beta}^{2}+\ell_{\bar{\gamma} \alpha}^{2} \ell_{\gamma \beta}\right) \\
& -\frac{1}{2}\left(\ell_{\alpha \beta}^{3}+\ell_{\beta \alpha}^{3}\right) \\
& +\varepsilon\left(|\ell|^{2} \ell_{\alpha \beta}-L^{N} \ell_{(\alpha \beta)}^{2}+i \sqrt{2} \ell_{\alpha \beta} L_{n}^{N}\right) \\
& \square_{\varepsilon} \ell_{n \alpha}=\left(\frac{\partial}{\partial t} \ell_{n \alpha}-2\left({ }^{H} \nabla^{2} K\right)\left(f_{n}, f_{\alpha}\right)\right)+\left(2\left({ }^{H} \nabla^{2} L\right)\left(f_{n}, f_{\alpha}\right)-{ }^{H} \Delta \ell_{n \alpha}\right) \\
& +\varepsilon\left(2\left({ }^{N} \nabla^{2} L^{N}\right)\left(f_{n}, f_{\alpha}\right)+2 L_{n}^{N}{ }^{N} \omega_{n}^{n}\left(f_{\alpha}\right)-{ }^{N} \Delta \ell_{n \alpha}\right) \\
& =i \sqrt{2}\left(\ell_{\alpha \gamma} K_{\bar{\gamma}}-\ell_{\alpha \bar{\gamma}} K_{\gamma}+\ell_{n n} K_{\alpha}-\ell_{\alpha n} K_{n}\right) \\
& +\frac{1}{2} K \ell_{\alpha \bar{\eta}} \ell_{\eta n}+K \ell_{\alpha n} \ell_{n n} \\
& +i \sqrt{2}\left[-2 \ell_{\alpha \bar{\gamma}} \ell_{n \gamma n}^{H}-2 \ell_{n n} L_{\alpha}-\ell_{\alpha \gamma} L_{\bar{\gamma}}\right. \\
& \left.+\ell_{\alpha \bar{\gamma}} L_{\gamma}+\frac{1}{2}\left(\ell_{\gamma \delta} \ell_{\bar{\delta} \bar{\gamma} \alpha}-\ell_{\bar{\gamma} \bar{\delta}} \ell_{\delta \gamma \alpha}\right)\right] \\
& +\ell_{n \alpha}\left(\ell_{\gamma \bar{\gamma}}^{2}+\ell_{\bar{\gamma} \gamma}^{2}\right)-\frac{1}{2} L\left(\ell_{n \alpha}^{2}+\ell_{\alpha n}^{2}+2 \ell_{n \alpha} \ell_{n n}\right) \\
& -2\left(\ell_{\alpha \bar{\gamma}} \ell_{n \gamma}^{2}+\ell_{n \alpha}^{3}\right)+\frac{1}{2}\left(\ell_{n \gamma} \ell_{\bar{\gamma} \alpha}^{2}+\ell_{\alpha n}^{3}\right) \\
& +\varepsilon\left(|\ell|^{2} \ell_{\alpha n}-L^{N} \ell_{(\alpha n)}^{2}\right)
\end{aligned}
$$




$$
\begin{aligned}
& \square_{\varepsilon} \ell_{n n}=\left(\frac{\partial}{\partial t} \ell_{n n}-2\left({ }^{H} \nabla^{2} K\right)\left(f_{n}, f_{n}\right)\right)+\left(2\left({ }^{H} \nabla^{2} L\right)\left(f_{n}, f_{n}\right)-{ }^{H} \Delta \ell_{n n}\right) \\
& +\varepsilon\left(2\left({ }^{N} \nabla^{2} L^{N}\right)\left(f_{n}, f_{n}\right)+2 L_{n}^{N}{ }^{N} \omega_{n}^{n}\left(f_{n}\right)-{ }^{N} \Delta \ell_{n n}\right) \\
& =\frac{3 i}{\sqrt{2}}\left(\ell_{n \gamma} K_{\bar{\gamma}}-\ell_{n \bar{\gamma}} K_{\gamma}\right)+\frac{1}{2} K \ell_{n \bar{\eta}} \ell_{\eta n}+K\left(\ell_{n n}\right)^{2} \\
& +i \sqrt{2}\left[\ell_{n \gamma} \ell_{n n \bar{\gamma}}-\ell_{n \bar{\gamma}} \ell_{n n \gamma}+\frac{3}{2}\left(\ell_{n \bar{\gamma}} L_{\gamma}-\ell_{n \gamma} L_{\bar{\gamma}}\right)\right. \\
& \left.+\ell_{\gamma \delta} \ell_{\bar{\gamma} \bar{\delta} n}-\ell_{\bar{\gamma} \bar{\delta}} \ell_{\gamma \delta n}\right] \\
& +\frac{3}{2} \ell_{n n}\left(\ell_{\gamma \bar{\gamma}}^{2}+\ell_{\bar{\gamma} \gamma}^{2}+\frac{2}{3} \ell_{n n}^{2}\right)-L\left(\ell_{n n}^{2}+\left(\ell_{n n}\right)^{2}\right) \\
& -6 \ell_{n n}^{3}+\frac{1}{2}\left(\ell_{\gamma \bar{\delta}} \ell_{\bar{\gamma} \delta}^{2}-\ell_{\gamma \bar{\gamma}}^{3}\right)+\varepsilon\left(|\ell|^{2} \ell_{n n}-L^{N} \ell_{(n n)}^{2}\right) .
\end{aligned}
$$

Further simplification immediately leads to the claim.

The above Proposition implies the following estimate.

Proposition 3.5. Let $0 \leq \varepsilon \leq 1$. Then there is a constant $c_{0}$ depending only on $n$ such that

$$
\square_{\varepsilon}|\ell|^{2} \leq-\left.\left.2\right|^{H} \nabla_{H} \ell\right|^{2}+c_{0}|\ell|^{4} .
$$

Proof. For two tensors $S, T$ we denote by $S * T$ any tensor whose entries are homogeneous polynomials of degree two and are linear in both $S$ and $T$. We set $\nabla={ }^{N} \nabla$, then we denote by $\nabla_{H}$ the covariant derivative taken in direction $H N$. Similarly for the connection ${ }^{H} \nabla$, we have ${ }^{H} \nabla_{H}$ standing for the covariant derivative w.r.t. ${ }^{H} \nabla$, taken in direction $H N$. With this notation, we have $\left(\nabla-{ }^{H} \nabla\right) T=\ell * T$, and Proposition 3.4 gives

$$
\square_{\varepsilon} \ell_{p a}=\ell *{ }^{H} \nabla_{H} \ell+\ell * \ell * \ell+\varepsilon(\ell * \nabla \ell+\ell * \ell * \ell) .
$$

Here we also used the Codazzi-type commutator relation [K, 2.4] to replace ${ }^{H} \nabla L$ by ${ }^{H} \nabla_{H} \ell+\ell * \ell$. Therefore we have for any pair of indices $(p a)$

$$
\begin{array}{r}
\frac{\partial}{\partial t}\left|\ell_{p a}\right|^{2}=\left({ }^{H} \Delta+\varepsilon^{N} \Delta\right)\left|\ell_{p a}\right|^{2}-2\left|{ }^{H} \nabla_{H} \ell_{p a}\right|^{2}+\ell * \ell *{ }^{H} \nabla_{H} \ell+\ell * \ell * \ell * \ell \\
+\varepsilon\left(-2\left|\nabla \ell_{p a}\right|^{2}+\ell * \ell * \nabla \ell+\ell * \ell * \ell * \ell\right) .
\end{array}
$$

Now using Cauchy-Schwarz, this completes the proof.

Corollary 3.6. If at $t=0$ we have a curvature bound of the form $\max _{N_{0}}|\ell|^{2} \leq$ $B$, then $\max _{N_{t}}|\ell|^{2} \leq \frac{B}{1-c_{0} B t}$. In particular, the curvature will not blow up for $t \in\left[0, c_{0}^{-1} B^{-1}\right)$.

Proof. Since $\square_{\varepsilon}$ is a (weakly) parabolic operator for $0 \leq \varepsilon \leq 1$, we can apply the parabolic maximum principle to compare with the solution of the ODE $\dot{g}=c_{0} g^{2}$ with initial value $B$, which is given by $g(t)=\frac{B}{1-c_{0} B t}$. 
We will also need to control higher order derivatives and set

$$
\left|\nabla^{m} \ell\right|^{2}=\sum_{p, a}\left|\nabla^{m} \ell_{p a}\right|^{2}
$$

Proposition 3.7. Let $0 \leq \varepsilon \leq 1$.

(i) There are constants $c_{1}$ and $c_{2}$ depending only on $n$ such that the first derivatives of the curvature satisfy the estimate

$$
\square_{\varepsilon}|\nabla \ell|^{2} \leq-\left|{ }^{H} \nabla_{H} \nabla \ell\right|^{2}+c_{1}|\nabla \ell|^{3}+c_{2}|\ell|^{2}|\nabla \ell|^{2} .
$$

(ii) For each $m \geq 2$ there are constants $c(n, m)$ depending only on $n, m$ and $\max _{N \times[0, T)}\left|\nabla^{i} \ell\right|$ for $0 \leq i \leq m-1$ such that

$$
\square_{\varepsilon}\left|\nabla^{m} \ell\right|^{2} \leq-\left.\left.\right|^{H} \nabla_{H} \nabla^{m} \ell\right|^{2}+c(n, m)\left(1+\left|\nabla^{m} \ell\right|^{2}\right) .
$$

Proof. Since the good Bochner-type term on the RHS will look like $-\left.{ }^{H} \nabla_{H} \nabla^{m-1} \ell\right|^{2}$, which doesn't dominate the total $m$-th derivative of $\ell$, we need to keep track of the horizontal derivatives in the subsequent computations.

We compute

$$
\left(\nabla_{\partial / \partial t} \nabla_{f_{a}}-\nabla_{f_{a}} \nabla_{\partial / \partial t}-\nabla_{\left[\partial / \partial t, f_{a}\right]}\right) f_{b}=\left(\nabla_{\partial / \partial t} \theta^{c}\right)\left(\nabla_{f_{a}} f_{b}\right) f_{c} .
$$

By (3.2),(3.3),(3.6) and (3.7), the coefficients of the RHS are polynomials of type $\ell * \nabla \ell$. By (3.6), (3.7), the third term on the LHS is of type $(\ell * \ell+\nabla \ell) * \nabla f_{b}$. (Note that the term $\nabla \ell$ does not occur in the mean curvature flow, where the frame is unrestrained in tangential directions. This additional term in the Levi flow causes the cubic term in the equation for the gradient above.) We conclude, for a tensor $T$,

$$
\left(\nabla_{\partial / \partial t} \nabla_{f_{a}}-\nabla_{f_{a}} \nabla_{\partial / \partial t}\right) T=\ell * \nabla \ell * T+(\ell * \ell+\nabla \ell) * \nabla T
$$

such that in particular

$$
\begin{aligned}
&\left(\nabla_{\partial / \partial t} \nabla^{m}-\nabla^{m} \nabla_{\partial / \partial t}\right) \ell_{p a}= \sum_{r=0}^{m-1} \nabla^{r}\left(\nabla_{\partial / \partial t} \nabla-\nabla \nabla_{\partial / \partial t}\right) \nabla^{m-r-1} \ell \\
&= \sum_{r=0}^{m-1} \nabla^{r}\left[\ell * \nabla \ell * \nabla^{m-r-1} \ell\right. \\
&=\left.+\ell * \ell * \nabla^{m-r} \ell+\nabla \ell * \nabla^{m-r} \ell\right] \\
& \sum_{i+j+k=m} \nabla^{i} \ell * \nabla^{j} \ell * \nabla^{k} \ell \\
&+\sum_{\substack{i+j=m+1 \\
i, j \geq 1}} \nabla^{i} \ell * \nabla^{j} \ell .
\end{aligned}
$$

We also need commutator relations on $\mathbb{C} \otimes T N$. By the Gauss-type equation in $[\mathrm{K}, 2.1]$, we have ${ }^{N} \Omega=\ell * \ell$. Also, when expressed as a linear combination of $\left\{f_{c}\right\}$, the vector $\left[f_{a}, f_{b}\right]$ has coefficients of type $\ell$. This gives $\left(\nabla_{f_{a}} \nabla_{f_{b}}-\nabla_{f_{b}} \nabla_{f_{a}}\right) T=$ $\ell * \nabla T+\ell * \ell * T$. 
In addition, since $\left(\nabla-{ }^{H} \nabla\right) T=\ell * T$, we have

$$
\begin{aligned}
{ }^{H} \Delta T & =\left(\nabla_{H}+{ }^{H} \nabla_{H}-\nabla_{H}\right)^{2} T \\
& =\nabla_{H}^{2} T+\nabla_{H} \ell * T+\ell * \nabla_{H} T+\ell * \ell * T .
\end{aligned}
$$

Therefore we compute the commutator of the horizontal Laplacian

$$
\begin{aligned}
\left(\nabla^{m}{ }^{H} \Delta-{ }^{H} \Delta \nabla^{m}\right) \ell_{p a} & =\left(\nabla^{m} \nabla_{H}^{2}-\nabla_{H}^{2} \nabla^{m}\right) \ell+\nabla^{m}\left(\ell * \nabla_{H} \ell+\ell * \ell * \ell\right) \\
& =\sum_{r=0}^{m-1} \nabla^{r}\left(\nabla_{H} \nabla-\nabla \nabla_{H}\right) \nabla^{m-r-1} \nabla_{H} \ell \\
& +\nabla^{m}\left(\ell * \nabla_{H} \ell+\ell * \ell * \ell\right) \\
& =\sum_{r=0}^{m-1} \nabla^{r}\left(\ell * \nabla^{m-r} \nabla_{H} \ell+\ell * \ell * \nabla^{m-r-1} \nabla_{H} \ell\right) \\
& =\sum_{i+j=m} \nabla^{i} \ell * \nabla^{j} \nabla_{H} \ell+\sum_{i+j+k=m} \nabla^{i} \ell * \nabla^{j} \ell * \nabla^{k} \ell \\
& =\sum_{i+j=m} \nabla^{i} \ell *{ }^{H} \nabla_{H} \nabla^{j} \ell+\sum_{i+j+k=m} \nabla^{i} \ell * \nabla^{j} \ell * \nabla^{k} \ell .
\end{aligned}
$$

Using then

$$
\begin{aligned}
\left(\nabla^{m}{ }^{N} \nabla_{f_{a}}^{N} \nabla_{f_{a}}-{ }^{N} \nabla_{f_{a}}^{N} \nabla_{f_{a}} \nabla^{m}\right) \ell_{p a} & =\sum_{r=0}^{m-1} \nabla^{r}\left(\nabla_{f a} \nabla-\nabla \nabla_{f a}\right) \nabla^{m-r} \ell \\
& =\sum_{r=0}^{m-1} \nabla^{r}\left(\ell * \nabla^{m-r+1} \ell+\ell * \ell \times \nabla^{m-r} \ell\right),
\end{aligned}
$$

we conclude for the Riemannian Laplacian

$$
\left(\nabla^{m}{ }^{N} \Delta-{ }^{N} \Delta \nabla^{m}\right) \ell=\sum_{i+j=m+1} \nabla^{i} \ell * \nabla^{j} \ell+\sum_{i+j+k=m} \nabla^{i} \ell * \nabla^{j} \ell * \nabla^{k} \ell
$$

Now, by Proposition 3.4 and the Codazzi equations established in [K,2.4], we may write the evolution equations of the curvature as

$$
\frac{\partial}{\partial t} \ell_{p a}=\left({ }^{H} \Delta+\varepsilon{ }^{N} \Delta\right) \ell_{p a}+\ell *{ }^{H} \nabla_{H} \ell+\ell * \ell * \ell
$$


Combining these identities we obtain for the higher derivatives of the curvature

$$
\begin{aligned}
\frac{\partial}{\partial t}\left|\nabla^{m} \ell_{p a}\right|^{2}=\left[\left(\nabla_{\partial / \partial t}\right.\right. & \left.\nabla^{m}-\nabla^{m} \nabla_{\partial / \partial t}\right) \ell_{p a} \\
& +\nabla^{m}\left({ }^{H} \Delta \ell_{p a}+\ell *{ }^{H} \nabla_{H} \ell+\ell * \ell * \ell\right) \\
& \left.+\varepsilon \nabla^{m}{ }^{N} \Delta \ell_{p a}\right] \nabla^{m} \ell_{\overline{p a}} \\
& + \text { complex conjugate } \\
=\quad\left({ }^{H} \Delta+\right. & \left.\varepsilon^{N} \Delta\right)\left|\nabla^{m} \ell_{p a}\right|^{2}-\left.\left.2\right|^{H} \nabla_{H} \nabla^{m} \ell_{p a}\right|^{2}-2 \varepsilon\left|\nabla^{m+1} \ell_{p a}\right|^{2} \\
& +\left(\nabla_{\partial / \partial t} \nabla^{m}-\nabla^{m} \nabla_{\partial / \partial t}\right) \ell * \nabla^{m} \ell \\
& +\left(\nabla^{m}{ }^{H} \Delta-{ }^{H} \Delta \nabla^{m}\right) \ell * \nabla^{m} \ell \\
& +\nabla^{m}\left(\ell *{ }^{H} \nabla_{H} \ell+\ell * \ell * \ell\right) * \nabla^{m} \ell \\
& +\varepsilon\left(\nabla^{m}{ }^{N} \Delta-{ }^{N} \Delta \nabla^{m}\right) \ell * \nabla^{m} \ell .
\end{aligned}
$$

Hence we finally conclude, setting $\left|\nabla^{m} \ell\right|^{2}:=\sum_{p, a}\left|\nabla^{m} \ell_{p a}\right|^{2}$,

$$
\begin{aligned}
& \square_{\varepsilon}\left|\nabla^{m} \ell\right|^{2}=-\left.\left.2\right|^{H} \nabla_{H} \ell\right|^{2}+\sum_{i+j=m} \nabla^{i} \ell *{ }^{H} \nabla_{H} \nabla^{j} \ell * \nabla^{m} \ell \\
& +\sum_{\substack{i+j=m+1 \\
i, j \geq 1}} \nabla^{i} \ell * \nabla^{j} \ell * \nabla^{m} \ell+\sum_{i+j+k=m} \nabla^{i} \ell * \nabla^{j} \ell * \nabla^{k} \ell * \nabla^{m} \ell \\
& +\varepsilon\left(-2\left|\nabla^{m+1} \ell\right|^{2}+\sum_{i+j=m+1} \nabla^{i} \ell * \nabla^{j} \ell * \nabla^{m} \ell\right. \\
& \left.+\sum_{i+j+k=m} \nabla^{i} \ell * \nabla^{j} \ell * \nabla^{k} \ell * \nabla^{m} \ell\right) .
\end{aligned}
$$

Estimating the terms on the RHS by their absolute value and using $a b \leq$ $\delta a^{2}+\left(\delta^{-1} / 4\right) b^{2}$, we then derive

$$
\left|\ell *{ }^{H} \nabla_{H} \nabla^{m} \ell * \nabla^{m} \ell\right| \leq\left|{ }^{H} \nabla_{H} \nabla^{m} \ell\right|^{2}+c(n, m)|\ell|^{2}\left|\nabla^{m} \ell\right|^{2},
$$

such that

$$
\begin{aligned}
\square_{\varepsilon}\left|\nabla^{m} \ell\right|^{2} \leq-\left|{ }^{H} \nabla_{H} \ell\right|^{2} & +c(n, m)\left(|\ell|^{2}+|\nabla \ell|\right)\left|\nabla^{m} \ell\right|^{2} \\
& +c(n, m) \sum_{\substack{i+j=m \\
i \leq m-1, j \leq m-2}}\left|\nabla^{i} \ell\right|^{2}\left|\nabla^{j+1} \ell\right|^{2} \\
& +c(n, m) \sum_{\substack{i+j+k=m \\
i, j, k \leq m-1}}\left|\nabla^{i} \ell\right|^{2}\left|\nabla^{j} \ell\right|^{2}\left|\nabla^{k} \ell\right|^{2} .
\end{aligned}
$$

Corollary 3.8. (i) Let $D=\max _{N_{0}}|\nabla \ell|^{2}$ and $0 \leq \varepsilon \leq 1$. There are constants $c_{3}>0, c_{4}>0$ depending only on $c_{0}(n), c_{1}(n), c_{2}(n)$ defined 
previously such that $\max _{N_{t}}|\nabla \ell|^{2} \leq 2 \max \left\{D, B^{2}\right\}$ for all $0 \leq t \leq t_{1}:=$ $\min \left\{c_{3} B^{-1}, c_{4} D^{-1 / 2}\right\}$.

(ii) For each $m \geq 2$ there is a constant $c_{m}$ depending on $n, m$ and $\max _{N_{0}}\left|\nabla^{i} \ell\right|^{2}$, $1 \leq i \leq m$, such that $\max _{N_{t}}\left|\nabla^{m} \ell\right|^{2} \leq c_{m}$ for all $0 \leq t \leq t_{1}$.

Proof. (i) By Proposition 3.7 we have

$$
\begin{aligned}
\square_{\varepsilon}|\nabla \ell|^{2} & \leq c_{1}|\nabla \ell|^{3}+c_{2}|\ell|^{2}|\nabla \ell|^{2} \\
& \left.\leq\left(c_{1}+c_{2}\right)\right)|\nabla \ell|^{3}+\frac{c_{2} B^{3}}{\left(1-c_{0} B t\right)^{3}},
\end{aligned}
$$

by Young's inequality and Corollary 3.6. Then $|\nabla \ell|^{2}$ is dominated by the comparison function $g(t)=k_{1}^{2} /\left(1-k_{2} t\right)^{2}$ if $k_{1}^{2}=\max \left\{D, B^{2}\right\}, k_{2}=\max \left\{\left(2 c_{0}+\right.\right.$ $\left.\left.2 \sqrt{c_{2}}\right) B, \sqrt{c_{1}+c_{2}} k_{1}\right\}$ and $t_{1}=\min \left\{c_{3} B^{-1}, c_{4} D^{-\frac{1}{2}}\right\}$ is chosen small enough. This gives the desired estimate.

(ii) The higher derivative estimates follow from induction and Proposition 3.7(ii), since the interval is bounded.

Lemma 3.9. Let $0 \leq \varepsilon \leq 1$. Then for any solution of $(d / d t) F=(L+$ $\left.\varepsilon L^{N}\right) X_{2 n}$, the induced metric ${ }^{N} h$ on the hypersurfaces $N_{t}$ is uniformly equivalent to the initial metric on $N_{0}$ for $0 \leq t \leq 1 / 2 t_{0}$.

Proof. This follows from the boundedness of $|\ell|^{2}$ for $0 \leq t \leq 1 / 2 t_{0}$ as in [Ha] since $|\ell|^{2}$ controls the derivative of the induced metric in view of (2.1).

Proof of Theorem 3.1. Since the evolution equation $(d / d t) F=\left(L+\varepsilon L^{N}\right) X_{2 n}$ is strictly parabolic up to tangential diffeomorphisms for each $0<\varepsilon \leq 1$, there is a smooth solution $F^{\varepsilon}$ with initial data $F_{0}$ for $0<\varepsilon \leq 1$. In view of the Corollary and Lemma, each solution $F^{\varepsilon}$ can be smoothly extended up to $t_{1}=$ $\min \left\{1 / 2 c_{0}^{-1} B^{-1}, c_{3} D^{-1 / 2}\right\}$. In view of these uniform estimates we may then pass $\varepsilon \rightarrow 0$ to obtain the desired solution of (1.1).

\section{Barrier principle}

We demonstrate the parabolic nature of the flow (1.1) by showing that the distance between two disjoint surfaces is nondecreasing under the flow. In addition, embedded surfaces remain embedded.

Theorem 4.1. (i) If $F_{j}: N^{j} \times[0, t) \rightarrow \mathbb{C}^{n}, j=1,2$, are two smooth solutions of (1.1) with $N_{0}^{1} \cap N_{0}^{2}=\emptyset$, then $N_{t}^{1} \cap N_{t}^{2}=\emptyset$ for all $t \in[0, T)$ and $\operatorname{dist}\left(N_{t}^{1}, N_{t}^{2}\right)$ does not decrease in time.

(ii) If $F_{0}: N \rightarrow \mathbb{C}^{n}$ is embedded, then the solution $F_{t}$ of (1.1) remains embedded for all $t \in[0, T)$.

Corollary 4.2. If $N_{0} \subset B\left(x_{0}, R\right)$ then $N_{t} \subset B\left(x_{0}, R_{t}\right)$ for $R_{t}=\left(R_{0}^{2}-4(n-\right.$ $1) t)^{1 / 2}$. In particular, $T<(4(n-1))^{-1} R_{0}^{2}$. 
Proof of Theorem 4.1. For two hypersurfaces $F_{j}: N \times[0, T) \rightarrow \mathbb{C}^{n}, j=1,2$, evolving according to (1.1), we define the distance $g: N \times N \times[0, T) \rightarrow \mathbb{R}$ by $g(p, q, t)=\left|F_{1}(p, t)-F_{2}(q, t)\right|^{2}$. Then

$$
\frac{\partial}{\partial t} g=2\left(F_{1}-F_{2}, \dot{F}_{1}-\dot{F}_{2}\right)=2\left(F_{1}-F_{2},{ }^{H} \Delta F_{1}-{ }^{H} \Delta F_{2}\right) .
$$

Now let $(p, q, t)$ be an arbitrary point of $N^{1} \times N^{2} \times[0, T)$, where $g(p, q, t) \leq$ $g(p, q, t)$ for all $(p, q) \in N^{1} \times N^{2}$. In view of [Ha], it is sufficient to show that $(d / d t) g(p, q, t) \geq 0$. In $(p, q, t)$ we have $\left(F_{1}-F_{2}\right) \| X_{2 n}$. Choose a unitary frame $\left\{e_{\alpha}^{1}\right\}_{1}^{n-1}$ in $H^{1,0} N_{t}^{1}$ at the point $F_{1}(p, t)$ and extend it parallel along the straight line from $F_{1}(p, t)$ to $F_{2}(p, t)$, where we get again a unitary frame $\left\{e_{\alpha}^{2}\right\}_{1}^{n-1}$ in $H^{1,0} N_{t}^{2}$. The first variation of $g$ in direction of $e_{\xi}^{1} \oplus e_{\xi}^{2}$ is then given by

$$
\delta g\left(e_{\xi}^{1} \oplus e_{\xi}^{2}\right)=2\left(F_{1}-F_{2},{ }^{N} \nabla_{e_{\xi}^{1}} F_{1}-{ }^{N} \nabla_{e_{\xi}^{2}} F_{2}\right),
$$

and the second variation is given by

$$
\delta^{2} g\left(e_{\xi}^{1}, e_{\xi}^{2}\right) \otimes\left(e_{\bar{\xi}}^{1}, e_{\bar{\xi}}^{2}\right)=2\left|e_{\xi}^{1}-e_{\xi}^{2}\right|^{2}+2\left(F_{1}-F_{2},{ }^{N} \nabla_{e_{\bar{\xi}}^{1}} e_{\xi}^{1}-{ }^{N} \nabla_{e_{\xi}^{2}} e_{\xi}^{2}\right) .
$$

Since $X_{2 n} \in F_{j}^{*} T \mathbb{C}^{n}$ is normal to $T N$,

$$
\left(X_{2 n},{ }^{N} \nabla_{e_{\bar{\xi}}^{j}} e_{\xi}^{j}\right)=\left(X_{2 n},{ }^{H} \nabla_{e_{\bar{\xi}}^{j}} e_{\xi}^{j}\right)=\left(X_{2 n},{ }^{H} \Delta F_{j}\right) .
$$

Therefore,

$$
\frac{\partial}{\partial t} g(p, q, t)=\delta^{2} g\left(e_{\xi}^{1} \otimes e_{\xi}^{2}\right) \geq 0 .
$$

This proves that the minimal distance between $N_{t}^{1}$ and $N_{t}^{2}$ does not decrease. To show the second part of the Theorem, note that from Theorem 3.1 we know that the solution remains smooth and regularly immersed as long as the curvature and its gradient remain bounded. By the argument above, $g$ cannot attain a new interior minimum off the diagonal, which is the only place where it is zero initially by assumption. Let $T>0$ be the maximal time of existence of $F_{t}$ and $\delta>0$ be arbitrary. Then a uniform curvature bound $|\ell|^{2} \leq B_{\delta}$ exists on $N \times[0, T-\delta)$ and $g$ is uniformly bounded below on the boundary of a suitable small neighborhood of the diagonal in $N \times N$ for $t \in[0, T-\delta)$. Thus $g$ remains nonzero off the diagonal on $[0, T-\delta)$. Since $\delta>0$ was arbitrary, the result follows.

\section{Maximum principles}

In order to establish qualitative properties of solution to (1.1) as well as their shape, we use the parabolic maximum principle for the operator $\frac{d}{d t}-{ }^{H} \triangle$ when applied to $L$ and $\ell$.

Theorem 5.1. (i) $-L \geq 0$ is preserved under the flow (1.1) 
(ii) If at time $t=0$ we have $-L \geq c_{1}>0$ then $-L$ has a lower bound given by

$$
\min _{N_{t}}(-L) \geq \frac{c_{1}}{\sqrt{1-t c_{1}^{2} /(n-1)}} .
$$

Proof. Since Proposition 3.4 implies

$$
\left(\frac{d}{d t}-{ }^{H} \triangle\right) L=i \sqrt{2}\left(\ell_{n \bar{\gamma}} e_{\gamma}-\ell_{n \gamma} e_{\bar{\gamma}}\right) \cdot L+\frac{1}{2} L\left(\ell_{\gamma \bar{\gamma}}^{2}+\ell_{\bar{\gamma} \gamma}^{2}+2 \ell_{n n}^{2}\right)
$$

the first result follows from the weak maximum principle for $\frac{d}{d t}-{ }^{H} \Delta$. Since $\ell_{\gamma \bar{\gamma}}^{2} \geq \frac{1}{2 n-2} L^{2}$, we have $\left(\frac{d}{d t}-{ }^{H} \Delta\right)(-L) \geq i \sqrt{2}\left(\ell_{n \bar{\gamma}} e_{\gamma}-\ell_{n \gamma} e_{\bar{\gamma}}\right) \cdot(-L)-\frac{1}{2 n-2} L^{3}$. By comparison with the solutions of the ODE

$$
\frac{d}{d t} f=\frac{1}{2 n-2} f^{3}, \quad f(0)=c_{0},
$$

the second part of the result follows. Note that equality holds on spheres.

While we only use a weak version of the maximum principle in the last result, we now prove a strict Hopf-type parabolic maximum principle for pseudoconvex surfaces in $\mathbb{C}^{2}$. As a result, equation (1.1) provides a canonical deformation of smooth weakly pseudoconvex surfaces into strictly pseudoconvex surfaces in $\mathbb{C}^{2}$.

Theorem 5.2. Let $F_{0}: N^{3} \rightarrow \mathbb{C}^{2}$ be a smooth closed weakly pseudoconvex hypersurface. Then the solution $F: N^{3} \times[0, T) \rightarrow \mathbb{C}^{2}$ of $(1.1)$ on a maximal time interval has strictly pseudoconvex surfaces $N_{t}^{3}$ for all $0<t<T$.

For the proof of Theorem 5.2 we need a Frobenius-type integrability theorem for certain distributions. Let $U$ be a smooth real $n$-manifold and $D^{m} \subset T U$ a smooth distribution in $T U$, or a subbundle of $T U$, with fibre dimension $m<n$, and fix $p \in U$. A piecewise regular real curve in $U$ is called a $D$-curve if its tangent spaces lie in $D$. Now $\Gamma(D)$ denotes the set of points in $U$ which can be connected with $p$ by a $D$-curve.

Theorem 5.3. Assume that $D$ is involutive on $\Gamma(D)$. Then in a neighborhood $U_{p}$ of $p$ there exist coordinates $w: U_{p} \longrightarrow \mathbb{R}^{n}, w(p)=0$, with $\operatorname{span}\left\{\partial / \partial w^{i}\right\}_{1}^{m}=$ $D$ on $\left\{w^{\ell}=0\right\}_{m+1}^{n}$.

Proof. The Theorem holds in the case that the fibre dimension $m$ of $D$ equals one. We will use induction to prove it for any $m \leq n-1$. Assume it holds for any distribution of fibre dimension $m-1$. Let $D=\operatorname{span}\left\{X_{i}\right\}_{1}^{m}$ and $u: U_{p} \longrightarrow \mathbb{R}^{n}$, $u(p)=0$, be coordinates with $\partial / \partial u^{1}=X_{1}$, and $D_{1}=\left\{X \in D: X u^{1}=0\right\} \subset D$. Now if $X, \tilde{X} \in D_{1}$, then $[X, \tilde{X}] \in D$ on $\Gamma(D)$ and $[X, \tilde{X}] u^{1}=0$. Therefore $D_{1} \subset T U$ is involutive on $\Gamma(D)$. Now let $U_{1}:=\left\{u^{1}=0\right\} \subset U_{p}$, then $D_{1} \mid$ $U_{1} \subset T U_{1}$ is involutive on $\Gamma\left(D_{1}\right) \cap U_{1}$. By induction hypothesis, on some $U_{2} \subset U_{1}$, there exist coordinates $\left(v^{2}, \cdots, v^{n}\right): U_{2} \rightarrow \mathbb{R}^{n-1}, v(p)=0$, with $\operatorname{span}\left\{\partial / \partial v^{i}\right\}_{2}^{m}=D_{1} \mid U_{2}$ on $\left\{v^{\ell}=0\right\}_{m+1}^{n}$. We can shrink $U_{p}$ to define a map

$$
\pi_{1}: U_{p} \rightarrow U_{2}, \quad \pi_{1}(q)=u^{-1} \circ\left(0, u^{2}, \cdots, u^{n}\right)(q),
$$


and new coordinates $w: U_{p} \longrightarrow \mathbb{R}^{n}, w(p)=0$, by $w^{1}=u^{1}, w^{j}=v^{j} \circ \pi$, $j=2, \ldots, n$. Since $\operatorname{span}\left\{Y_{1}:=X_{1}, Y_{i}:=X_{i}-\left(X_{i} \cdot u^{1}\right) X_{1}\right\}_{2}^{m}=D$, we have $\left[Y_{i}, Y_{j}\right]=z_{i j k} Y_{k}$ on $\Gamma(D)$ for functions $z_{i j k}: \Gamma(D) \rightarrow \mathbb{R}$ that are smooth on smooth segments of curves in $\Gamma(D)$. Note that $Y_{1} w^{\ell}=0$ for $\ell=m+1, \ldots, n$, so for $j=2, \ldots, m$, we have $Y_{1}\left(Y_{j} w^{\ell}\right)=\left[Y_{1}, Y_{j}\right] w^{\ell}$ and by definition of $z_{i j k}$ :

$$
Y_{1}\left(Y_{j} w^{\ell}\right)=z_{i j k}\left(Y_{k} \cdot w^{\ell}\right) \text { on } \Gamma(D) \text {. }
$$

Since $Y_{j} \in \operatorname{span}\left\{\partial / \partial v^{j}\right\}_{2}^{m}$ and $w^{\ell}=v^{\ell}$ on $U_{2}$,

$$
Y_{j} w^{\ell}=0 \text { on } U_{2} \text {. }
$$

Denote by $\gamma_{v^{2}, \ldots, v^{m}}$ the integral curve of $Y_{1}$ passing through $\left(v^{2}, \ldots, v^{m}, 0, \ldots, 0\right)$ $\in U_{2} \cap\left\{v^{\ell}=0\right\}_{m+1}^{n}$. Then $\gamma_{v^{2}, \ldots, v^{m}}$ is contained in $\Gamma(D)$. The above system of ODEs along $\gamma_{v^{2}, \ldots, v^{m}}$ for the functions $\left(Y_{j} \cdot w^{\ell}\right)$ with initial condition (IC) on $U_{2} \cap\left\{v^{\ell}=0\right\}_{m+1}^{n}$ has the unique solution $Y_{j} \cdot w^{\ell}=0$ on $\gamma_{v^{2}, \ldots, v^{m}}$. Therefore on $\left\{w^{\ell}=0\right\}_{m+1}^{n}, \operatorname{span}\left\{\partial / \partial w^{i}\right\}_{1}^{m}=\operatorname{span}\left\{Y_{i}\right\}_{1}^{m}$, and the latter equals $D$.

Proof of Theorem 5.2. Suppose there is $p_{0} \in N, t \geq 0$ such that $L\left(p_{0}, t_{0}\right)=0$. For coordinates $z_{1}=x_{1}+i x_{2}, z_{3}=x_{3}+i x_{4}$ of $\mathbb{C}^{2}$ let $C_{4}$ be the largest $x_{4}$-value where $L$ equals zero at $t=t_{0}$, i.e.,

$$
A:=\left\{q \in N \mid L\left(q, t_{0}\right)=0\right\} \subset\left\{x_{4} \leq C_{4}\right\}, \quad A \cap\left\{x_{4}=C_{4}\right\} \neq \emptyset .
$$

Let $p \in A \cap\left\{x_{4}=C_{4}\right\}$. Notice that the first order transport term in the evolution equation of $L$ as given in the proof of Theorem 5.1 involves only horizontal derivatives of $L$ and therefore has no component in the direction where ${ }^{H} \Delta$ is degenerate. The strict maximum principle for degenerate parabolic equations, see [B] and [A], then applies and shows that $L$ has to be zero at each point $\left(q, t_{0}\right)$ which can be reached from $\mathrm{p}$ by curves following a direction in $H N_{t_{0}}$, i.e., in $\Gamma_{p}\left(H N_{t_{0}}\right) \subset A$. In particular, $\mathcal{C} \subset\left\{x_{4} \leq C_{4}\right\}$ and $p \in \mathcal{C} \cap\left\{x_{4} \leq C_{4}\right\}$. By the elliptic strong maximum principle for holomorphic curves it follows that $\mathcal{C}$ $\subset\left\{x_{4}=C_{4}\right\} \cap N_{t_{0}}$ and is a subset of an affine complex line in $\mathbb{C}^{2}$. Since $A$ and $N_{t_{0}}$ are closed, we could repeat the above argument at a possible boundary point of $\mathcal{C}$, concluding that $\mathcal{C}$ can be extended in $N_{t_{0}}$ to the entire affine complex line. This contradicts the compactness of $N_{t_{0}}$.

\section{References}

[A] S. Altschuler, A geometric heat flow for one-forms on three dimensional manifolds, Illinois J. Math. 39 (1995), 98-118.

[B] J.M. Bony, Principe du maximum, inégalité de Harnack, et unicité du problème de Cauchy pour les operateurs elliptiques degénérés, Ann. Inst. Fourier 19 (1969), 277304.

[C] G. Citti, $C^{\infty}$ regularity of solutions of the Levi equation, Ann. Inst. H. Poincaré Anal. non Linéaire 15 (1998), 517-534.

[CM] G. Citti and A. Montanari, Strong solutions for the Levi curvature equations, preprint 1999.

[Ha] R. Hamilton, Three-manifolds with positive Ricci curvature, J. Differential Geom. 17 (1982), 255-306. 
[H] G. Huisken, Flow by mean curvature of convex of convex surfaces into spheres, J. Differential Geom. 20 (1984), 237-266.

[K] W. Klingenberg, Real hypersurfaces in Kähler manifolds, to appear in Asian J. Math.

[PW] M. Protter and H. Weinberger, Maximum principles in differential equations, PrenticeHall, Inc., Englewood Cliffs, N.J., 1967.

[ST] Z. Slodkowski and G. Tomassini, Evolution of subsets in $C^{2}$ and a parabolic problem for the Levi equation, 1997, to appear in Ann. Scuola Norm. Sup. Pisa Cl. Sci.

Mathematisches Institut, Universität Tübingen, Auf Der Morgenstelle 10, 72076 TÜBINGEN, GERMANY

E-mail address: gerhard.huisken@uni-tuebingen.de

Institut für Reine Mathematik, Humboldt Universität Zu Berlin, Ziegelstrasse 13A, 10099 BERLIN, GERMANY

E-mail address: klingenb@mathematik.hu-berlin.de 